\title{
SÍNDROME DE HOFFMAN: REPORTE DE UN CASO DE MIOPATÍA TIROIDEA
}

HOFFMAN SYNDROME: CASE REPORT OF THYROID MYOPATHY

\author{
SÍNDROME DE HOFFMAN: RELATÓRIO DE UM
}

CASO DE MIOPATIA TIREÓIDE

\section{GUSTAVO LAMA RIVERA, IVETTE GUZMÁN ADUM, MIGUEL FLOR RODRÍGUEZ, ROBERTO MARIANI CARRERA}

Hospital Luis Vernaza, Guayaquil, Ecuador

Resumen

El hipotiroidismo primario constituye el $95 \%$ de las insuficiencias tiroideas. Este se presenta con diversas manifestaciones, entre ellas la debilidad simétrica proximal e hipertrofia muscular conocida como síndrome de Hoffman. Dicha patología se reporta únicamente en el $10 \%$ de casos, por lo que realizar el diagnóstico diferencial de debilidad muscular es indispensable. A continuación se relata el caso de un paciente de 69 años que consulta por debilidad muscular proximal progresiva, mialgias, dislalia y somnolencia; durante su hospitalización fue diagnosticado con tiroiditis de Hashimoto y miopatía hipotiroidea hipertrófica del adulto.

PALABRAS CLAVE: hipotiroidismo, miopatía, Hoffman, atrofias musculares espinales de la infancia.

Abstract

Primary hypothyroidism constitutes $95 \%$ of thyroid insufficiencies. This present with various manifestations, including proximal symmetric weakness and muscle hypertrophy known as Hoffman's syndrome. This pathology is only reported in $10 \%$ of cases, so making a differential diagnosis of muscle weakness is essential. We report the case of a 69 -year-old patient who consulted for progressive proximal muscle weakness, myalgia, dyslalia, and somnolence; During his hospitalization, he was diagnosed with Hashimoto's thyroiditis and adult hypertrophic hypothyroid myopathy.

KEYwORDS: hypothyroidism, myopathies, Hoffman, spinal muscular atrophies of childhood.

Resumo

O hipotireoidismo primário constitui 95\% das insuficiências da tireóide. Isso ocorre com várias manifestaç̃oes, incluindo fraqueza simétrica proximal e hipertrofia muscular conhecida como síndrome de Hoffman. Esta patologia é relatada apenas em $10 \%$ dos casos, sendo essencial o diagnóstico diferencial de fraqueza muscular. 0 seguinte é o caso de um paciente de 69 anos que consultou fraqueza muscular proximal progressiva, mialgia, dislalia e sonolência; Durante sua internação, ele foi diagnosticado com tireoidite de Hashimoto e miopatia por hipotireoidismo hipertrófico em adultos. PALABRAS-CHAVE: hipotireoidismo, miopatia, Hoffman, atrofias musculares espinhais da infância. 
INTRODUCCIÓN

Las hormonas tiroideas son indispensables para el metabolismo celular siendo el hipotiroidismo una enfermedad multisistémica de inicio insidioso con consecuencias severas a largo plazo. El hipotiroidismo primario constituye el $95 \%$ de las insuficiencias tiroideas. La principal etiología es la tiroiditis de Hashimoto, variedad de tiroiditis crónica autoinmune que se caracteriza por niveles elevados de anticuerpos anti-peroxidasa tiroidea (90-100\% mayor afinidad y mayores concentraciones) y anti-tiroglobulina (80-90\%).1, 2

En el hipotiroidismo puede presentarse una gran variedad de manifestaciones, entre las que se encuentran las miopatías, mononeuropatías y polineuropatías axonales sensitivo-motoras. La miopatía asociada con hipotiroidismo se puede dividir en 4 subtipos: el síndrome de KocherDebré-Semelaigne o miopatía hipertrófica congénita (pacientes pediátricos con cretinismo), el síndrome de Hoffman o miopatía hipertrófica del adulto, la forma atrófica y el síndrome miasténicos. ${ }^{1}$

El Síndrome de Hoffman es una forma infrecuente de afectación muscular que aparece en pacientes con hipotiroidismo grave de largo tiempo de evolución. Se caracteriza por debilidad simétrica proximal e hipertrofia. Fue descrita por primera vez en 1897, en un paciente con rigidez y dificultad para la relajación muscular luego de una tiroidectomía. ${ }^{2}$

El Síndrome de Hoffman, más allá de su baja prevalencia, entraña un reto diagnóstico importante. Ya que existen múltiples factores asociados a miopatías en los pacientes típicamente afectados por el síndrome. Además de que el hipotiroidismo severo usualmente se acompaña de deterioro del nivel de conciencia, lo que dificulta en gran medida el examen físico neurológico e incluso lleva a un segundo plano la debilidad muscular como motivo de consulta y parte indispensable del cuadro clínico.

\section{CASO CLÍNICO}

Paciente masculino de 69 años de edad con antecedentes patológicos personales de constipación tratada ocasionalmente con laxantes y consumo esporádico de alcohol. Acude a emergencia del hospital Luis Vernaza, por presentar cuadro clínico de aproximadamente dos meses de evolución, caracterizado por debilidad muscular proximal progresiva que se acompaña de mialgias en extremidades superiores e inferiores. Una semana antes de su admisión hospitalaria, se le dificulta la marcha, necesitando asistencia. Dos días previos a su ingreso, se le imposibilita la bipedestación; además presenta dislalia y somnolencia.

Al examen físico en el departamento de emergencia la presión arterial fue 100/60 $\mathrm{mmHg}$, pulso $54 \mathrm{lpm}$, frecuencia respiratoria $16 \mathrm{rpm}$, saturación de oxígeno 98\% al aire ambiente, temperatura $36.6^{\circ} \mathrm{C}$, peso $73 \mathrm{~kg}, 169$ centímetros de estatura. En la inspección la piel lucía seca y descamada con marcada alopecia. A la auscultación del tórax los ruidos cardíacos estaban bradicárdicos, rítmicos y sin soplos; campos pulmonares claros y ventilados. El abdomen se presentó distendido, con ruidos hidroaéreos disminuidos, blando, poco depresible, no doloroso, sin visceromegalias palpables y timpánico a la percusión. Las extremidades eran simétricas y sin edemas, con marcada hipertrofia muscular y dolor a la compresión en brazos, antebrazos, muslos y pantorrillas de ambos lados. (Figura 1).

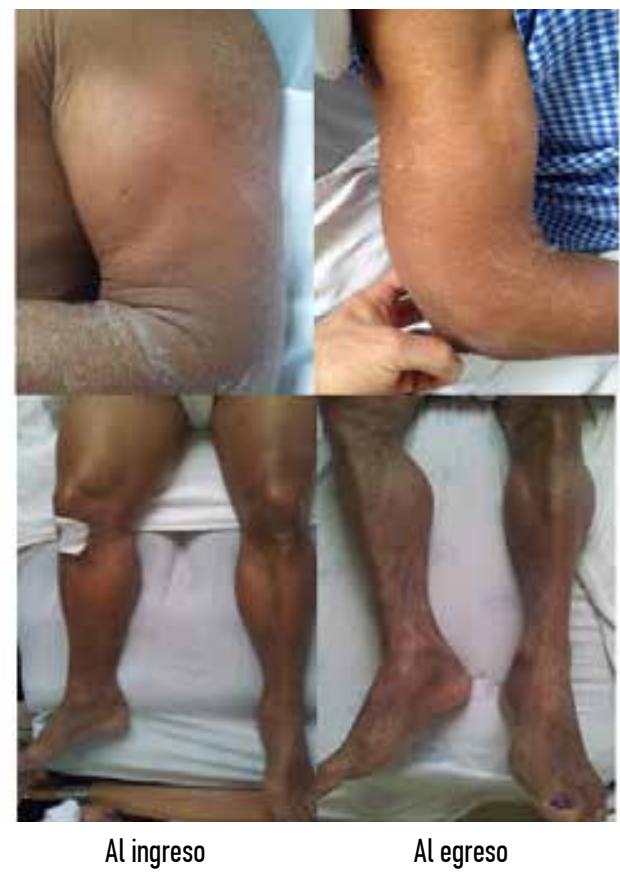

Figura 1. Hipertrofia muscular dolorosa en extremidades y su evolución durante la hospitalización.

A la exploración neurológica, se encontraba somnoliento y desorientado, con puntaje de 11/15 en la escala de Glasgow. Pupilas en $5 \mathrm{~mm}$, isocóricas reactivas. La motilidad pasiva era normal. Presentaba cuadriparesia proximal con fuerza muscular $2 / 5 \mathrm{y}$ marcada hipertrofia dolorosa a la compresión a nivel de extremidades, sin movimientos anormales. Reflejos osteoten- 
dinosos disminuidos. La sensibilidad superficial y profunda conservada. Lenguaje dislálico. Pares craneales, motilidad activa, taxia y praxia no pudieron ser examinados por su nivel de conciencia. Se solicitan laboratorios cuyos resultados se muestran en la tabla 1 , donde destaca la elevación de nitrogenados y transaminasas a predominio de TGO.

TABLA 1. DATOS DE LABORATORIO

\begin{tabular}{|c|c|c|c|c|}
\hline VARIABLE & $\begin{array}{l}\text { VALORES DE } \\
\text { REFERENCIA }\end{array}$ & AL INGRESO & DÍAS 2 - 4 & DÍA 35 \\
\hline Leucocitos & $4.40-10.0$ & 6.22 & & \\
\hline Neutrófilos & $50-70 \%$ & $78.2 \uparrow$ & & \\
\hline Linfocitos & $25-40 \%$ & $12.5 \downarrow$ & & \\
\hline Eritrocitos & $4.50-6.50$ & $3.32 \downarrow$ & & \\
\hline Hemoglobina & $13.5-17.3$ & $10.1 \downarrow$ & & \\
\hline Hematócrito & $40-52 \%$ & $30.8 \downarrow$ & & \\
\hline V.C.M. & $76.0-96.0$ & 92.8 & & \\
\hline H.C.M. & $28.0-33.0$ & 30.4 & & \\
\hline C.H.C.M. & $33-36.0$ & $32.8 \downarrow$ & & \\
\hline Plaquetas & $150-450$ & 245 & & \\
\hline Úrea & $16-48$ & $135 \uparrow$ & $133 \uparrow$ & 43 \\
\hline Creatinina & $0.50-1.30$ & $3.40 \uparrow$ & $2.89 \uparrow$ & 0.99 \\
\hline$\rightarrow$ MDRD & & 19.2 & & \\
\hline TGO & $0-38$ & $425 \uparrow$ & $260 \uparrow$ & $84 \uparrow$ \\
\hline TGP & $0-41$ & $150 \uparrow$ & $95 \uparrow$ & 36 \\
\hline Colinesterasa & $5320-12920$ & $4669 \downarrow$ & $3990 \downarrow$ & \\
\hline Sodio & $132-146$ & 132 & & 132 \\
\hline Potasio & $3.50-5.40$ & $5.50 \uparrow$ & 5.10 & 3.80 \\
\hline GGT & $8-61$ & & 38 & \\
\hline Fosfatasa alcalina & $40-129$ & & 104 & \\
\hline Bilirrubina total & $0-1$ & & 0.46 & \\
\hline Bilirrubina directa & $0-0.3$ & & 0.11 & \\
\hline Bilirrubina indirecta & $0-0.7$ & & 0.35 & \\
\hline Lipasa & $0-60$ & & $90 \uparrow$ & \\
\hline Amilasa & $28-100$ & & $139 \uparrow$ & \\
\hline Colesterol total & $0-200$ & & $283 \uparrow$ & \\
\hline Colesterol HDL & $35-80$ & & 45 & \\
\hline Colesterol LDL & $0-130$ & & $207 \uparrow$ & \\
\hline Proteínas totales & $6.50-8.00$ & & 6.65 & \\
\hline Albúmina & $3.50-5.00$ & & 3.63 & \\
\hline Globulina & $1.50-3.00$ & & 3.02 & \\
\hline LDH & $10-250$ & & $1810 \uparrow$ & $1796 \uparrow$ \\
\hline $\mathrm{CPK}$ & $0-190$ & & $8605 \uparrow$ & $1947 \uparrow$ \\
\hline Mioglobina & $16-76$ & & $608 \uparrow$ & $243 \uparrow$ \\
\hline $\mathrm{TSH}$ & $0.27-4.20$ & & $64.18 \uparrow$ & $27.91 \uparrow$ \\
\hline $\mathrm{T3}$ & $0.80-2.00$ & & $0.19 \downarrow$ & $0.56 \downarrow$ \\
\hline FT4 & $0.90-1.70$ & & $0.05 \downarrow$ & $0.52 \downarrow$ \\
\hline Cortisol AM & $2.3-11.9$ & & $14.1 \uparrow$ & \\
\hline Anti tiroperoxidasa & $0-34$ & & $98.6 \uparrow$ & \\
\hline Anti tiroglobulina & $0-115$ & & 20 & \\
\hline
\end{tabular}

Se realizó radiografía de tórax y tomografía simple de cerebro, sin hallazgos relevantes y el electrocardiograma reveló bradicardia sinusal y complejos QRS de bajo voltaje. (Figura 2).

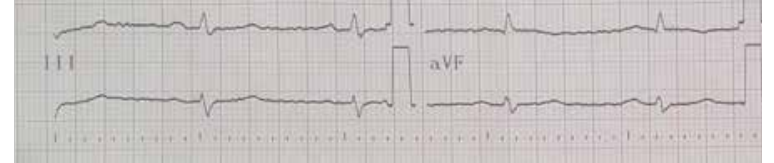

Figura 2. Electrocardiograma al ingreso hospitalario.

Tras la realización de pruebas complementarias en el abordaje diagnóstico del deterioro de nivel y contenido de conciencia, se certifica hipotiroidismo primario, hipercolesterolemia y elevación de enzimas musculares. Motivo por el cual se decide el ingreso hospitalario, con el fin de realizar estudios especializados, además de monitorizar su evolución y respuesta al tratamiento hormonal suplementario. En el segundo día de hospitalización, la ecografía de tiroides muestra lóbulos tiroideos atróficos. Se confirma la elevación de anticuerpos anti-peroxidasa tiroidea, con anti-tiroglobulina dentro de parámetros normales. Con los hallazgos confirmatorios de tiroiditis crónica autoinmune de Hashimoto en etapa avanzada (atrófica) y miopatía hipotiroidea hipertrófica, se llega al diagnóstico de Síndrome de Hoffman.

Se inicia tratamiento sustitutivo con levotiroxina $75 \mathrm{mcg} / \mathrm{día}$ que se incrementó progresivamente hasta llegar a $150 \mathrm{mcg} /$ día. Los niveles elevados de nitrogenados correspondían a una insuficiencia renal aguda por pigmentos (mioglobina), cuyos valores mejoraron notablemente con hidratación parenteral y diuréticos.

La evolución clínica del paciente se corresponde adecuadamente con la mejoría bioquímica demostrada en el seguimiento. Durante su hospitalización el nivel de conciencia mejora notablemente; de igual manera recupera fuerza muscular y se reduce el grado de hipertrofia en extremidades como muestra la figura 1. Es dado de alta 35 días después con Clasgow 14/15 y fuerza muscular 4/5. La TSH descendió, la T3 y fT4 se elevaron, pero sin entrar aún a rangos habituales. La CPK y la mioglobina también disminuyeron, aunque la LDH permaneció casi en el mismo nivel del ingreso. Hallazgos que son esperables por el tiempo de acción requerido con el uso de levotiroxina.

DISCUSIÓN

El síndrome de Hoffman es una entidad poco prevalente entre los pacientes con hipotiroidismo, pese a que hasta un $80 \%$ de los hipotiroideos presentan alguna forma de afectación muscular. La asociación de hipertrofia con debilidad 
proximal característica del Hoffman, se reporta en menos del $10 \%$ de los pacientes. Los mismos que típicamente, también refieren rigidez luego del ejercicio y dolor muscular en hombros y pelvis. Además de presentar enlentecimiento de sus funciones cognitivas y motoras. Los signos clásicos del síndrome son las mialgias, mixedema e hiporreflexia. ${ }^{1}$

La etiología de la hipertrofia muscular es motivo de discusión. Al parecer el déficit de hormona tiroidea lleva al paciente a un estado de resistencia a la insulina, ocasiona reducción de la glucogenólisis, de los receptores beta adrenérgicos y del metabolismo mitocondrial oxidativo, lo cual afecta la provisión de energía del músculo. Esto ocasiona dolor y atrofia selectiva de las fibras musculares tipo II (más dependientes de la glucólisis), con hipertrofia compensadora de las fibras tipo I, debido a la acumulación de glucosaminoglicanos.
El retraso en la contracción y relajación muscular puede deberse a la redistribución de fibras de contracción rápida a contracción lenta. ${ }^{3-4}$

Los grupos musculares más comúnmente involucrados son: la lengua y las extremidades superiores e inferiores. ${ }^{2}$ En la figura 1 se aprecia la hipertrofia muscular a nivel de brazo, muslos y pantorrillas del paciente al ingreso, y la disminución de volumen luego de 35 días de tratamiento sustitutivo hormonal.

Las manifestaciones de hipotiroidismo que llevan a distintos tipos de neuropatía y miopatía son responsables únicamente del 5\% de todas las miopatías adquiridas. ${ }^{4}$ Por lo que el diagnóstico diferencial debe incluir las principales causas de debilidad muscular, (figura 2).

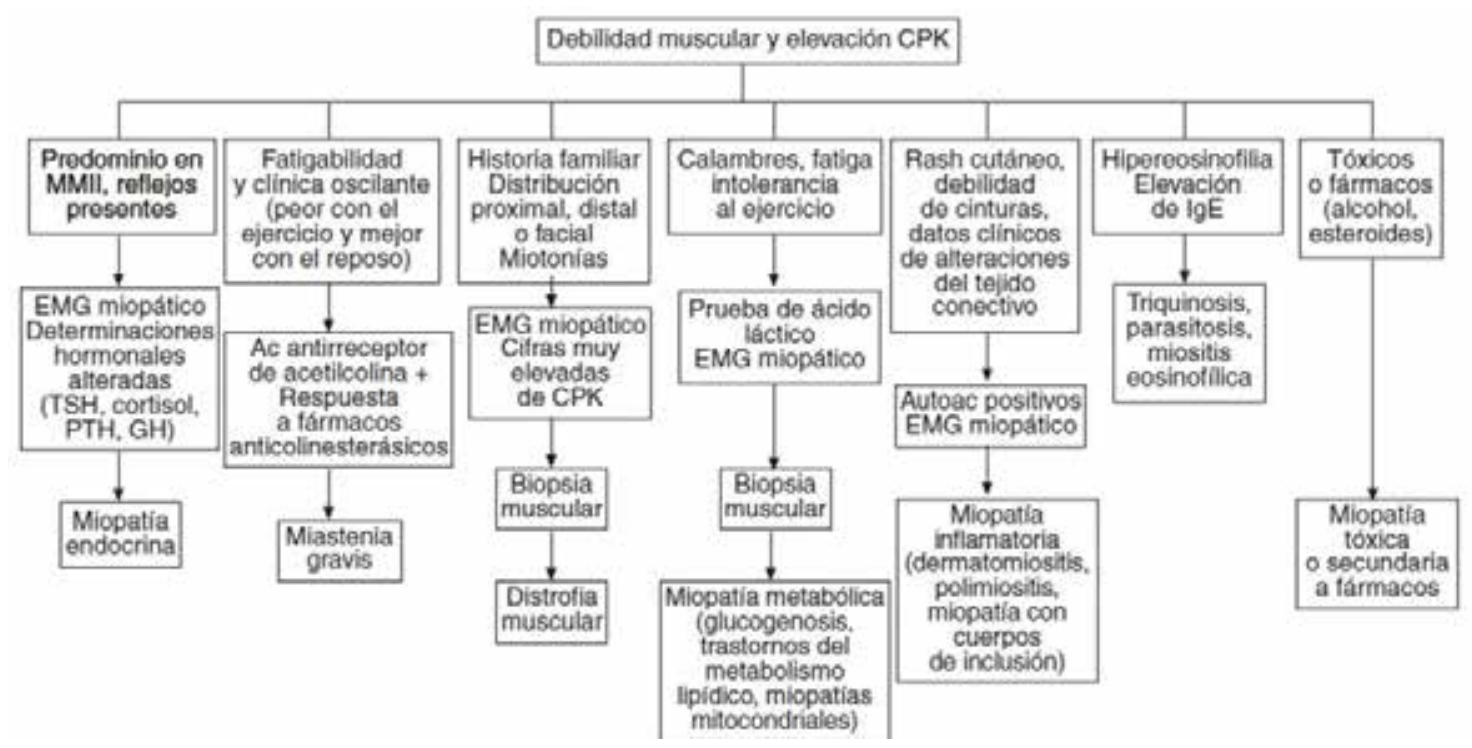

Figura 2. Algoritmo diagnóstico ante un paciente con debilidad muscular y elevación de la CPK ${ }^{6}$. Bouzas $E$ et al. debilidad e hipertrofia muscular como forma de presentación del hipotiroidismo: Síndrome de Hoffman. Semergen. 2008; 34(10):519-21.

Dicho diferencial inicia a partir de las enzimas musculares, especialmente la creatinfosfoquinasa (CPK), que es el mejor marcador bioquímico de miopatías. Estas suelen estar elevadas (80\% de casos), aunque no siempre se correlacionan con la severidad de los síntomas miopáticos. ${ }^{1}$ Usualmente en la miopatía tiroidea alcanza valores hasta seis veces el límite superior de la normalidad; valores mayores, hacen muy difícil su diferenciación con polimiositis y otros tipos de miopatía. La TGO,
TGP, LDH y aldolasa tienen únicamente un rol complementario en la investigación de esta patología..$^{1-5}$

La electromiografía (EMG) no suele ser concluyente, los hallazgos pueden ser compatibles con un patrón miogénico (disminución de la duración y amplitud de potenciales de unidad motora) o revelar alteraciones en la conducción nerviosa compatibles con mononeuropatía o polineuropatía sensitivomotora. ${ }^{2}$ 
El reemplazo con levotiroxina se realiza a razón de 100 a $200 \mathrm{mcg} / \mathrm{día}$, y permite la recuperación de las alteraciones bioquímicas y clínicas. Las enzimas musculares retornan a la normalidad luego de semanas de tratamiento, incluso antes que la TSH recupere sus valores habituales. ${ }^{6} \mathrm{La}$ debilidad muscular se recupera más lentamente a lo largo de meses o incluso años. ${ }^{1-7}$ Sin embargo, aproximadamente el 50\% de los pacientes tienen anomalías residuales persistentes si son rebiopsiados luego de la restitución hormonal. ${ }^{5}$

Se debe puntualizar que la biopsia muscular no es una técnica habitual en nuestro medio para el diagnóstico de miopatía tiroidea. Debido a los altos costos y poca disponibilidad, se limita a cuadros de diagnóstico incierto o con fines académicos. Una vez comprobado bioquímicamente el hipotiroidismo severo con clínica compatible e hipertrofia muscular (en el caso de Síndrome de Hoffman), se inicia el reemplazo hormonal con excelente respuesta al tratamiento a mediano y largo plazo.

Es indispensable la valoración del riesgo cardiovascular individual previo al inicio del tratamiento, especialmente en personas añosas. Se ha descrito un aumento en el riesgo de desarrollar insuficiencia coronaria aguda en estos pacientes debido al aumento de demanda metabólica. Adicionalmente, un porcentaje refiere progresión sintomática inicial, en cuyo caso las complicaciones más serias son la rabdomiólisis y debilidad muscular respiratoria, por lo que se recomienda el uso de corticoides debido a su efecto estabilizador de membrana. ${ }^{1,5,8}$
CONCLUSIÓN

El síndrome de Hoffman es una rara forma de miopatía hipertrófica presente en el hipotiroidismo, por lo que, ante el desarrollo de debilidad muscular con niveles elevados de CPK, se recomienda descartar una disfunción tiroidea.

\section{REFERENCIAS BIBLIOGRÁFICAS}

1. Vasconcellos L, Peixoto $M$, Nunes de Oliveira T, Penque G, Celestino A. Hoffman's Syndrome: Pseudohypertrophic Myopathy as Initial Manifestation of Hypothyroidism - Case Report. Arq Neuropsiquiatr. 2003;61(3-B):851-854.

2. Praveen KA, Aslam S, Dutta TK. Hoffmann's Syndrome: A Rare Neurological Presentation of Hypothyroidism. Int J Nutr Pharmacol Neurol Dis. 2011;1(2):201.

3. Senanayake H, Dedigama A, Alwis R, Thirumavalavan K. Hoffman Syndrome: A Case Report. Int Arch Med. 2014; 7(1):2.

4. Kiran HS, Murthy KAS, Aparna AN. A Young Lady with Swelling and Stiffness of Calf Muscles. Indian JEndocr Metab. 2011;15(2):130-131.

5. Shams N, Niaz F, Osmani H. Hoffman Syndrome: Rare Presentation of Hypothyroidism. JLUMHS. 2013; 12(03): 214-217.

6. Bouzas E, Menéndez JL, Cerrada E, López C, Ruiz M. Debilidad e hipertrofia muscular como forma de presentación de hipotiroidismo: síndrome de Hoffman. Semer-Med Fam. 2008;34(10):519-21.

7. Sundarachari N, Sridhar A. Lakshmi V. Rare Yet Treatable: Hypothyroid Myopathy (Hoffman Syndrome). J Dr NTR Univ Heal Sci 2013: 2(3):203-204.

8. Uddin MN, Uddin A, Chy MAU, Khan T. Hoffmann's Syndrome a Presenting Manifestation of $\mathrm{Hy}^{-}$ pothyroidism. Am J Med Case Reports. 2014; 2(4): 75-77. 